\title{
Article \\ Adaptive Impedance Matching Scheme for Magnetic MIMO Wireless Power Transfer System
}

\author{
Ziyang Lu ${ }^{1} \oplus$, Yubin Zhao ${ }^{1}$ and Dunge Liu ${ }^{2, *}$ \\ 1 School of Microelectronics Science and Technology, Sun Yat-sen University, Zhuhai 519082, China; \\ luzy3@mail2.sysu.edu.cn (Z.L.); zhaoyb23@mail.sysu.edu.cn (Y.Z.) \\ 2 State Key Laboratory of Space-Ground Integrated Information Technology, Space Star Technology Co., Ltd., \\ Beijing 100041, China \\ * Correspondence: zkydunge@163.com
}

Citation: Lu, Z.; Zhao, Y.; Liu, D Adaptive Impedance Matching Scheme for Magnetic MIMO Wireless Power Transfer System. Electronics 2021, 10, 2788. https://doi.org/ 10.3390 /electronics10222788

Academic Editor: Rafael González Ayestarán

Received: 15 September 2021 Accepted: 3 November 2021 Published: 14 November 2021

Publisher's Note: MDPI stays neutra with regard to jurisdictional claims in published maps and institutional affiliations.

Copyright: (c) 2021 by the authors. Licensee MDPI, Basel, Switzerland. This article is an open access article distributed under the terms and conditions of the Creative Commons Attribution (CC BY) license (https:// creativecommons.org/licenses/by/ $4.0 /)$.

\begin{abstract}
In coupled magnetic resonance (CMR) wireless energy transfer systems, the energy transfer power is low and the power transfer efficiency changes with the coil position. One reason for this reduction in power and efficiency is the impedance mismatching (IM) between the Tx and Rx coils; achieving impedance matching for multiple-input multiple-output (MIMO) CMR IM wireless power transmission (WPT) is quite complex due to the uncertainty in the number of coils and the interaction between coils. In this paper, we provide an analytical model of MIMO CMR which fully formulates the complex relationship between multiple Tx and Rx channels. Then, we design an impedance matching network (IMN) for MIMO CMR and derive an optimal IM solution. Base on this solution, we also develop an adaptive impedance matching scheme to control IMN, based on an automatic analysis of MIMO CMR system; the resulting control scheme achieves optimal values for transmission power and efficiency through IMN and coil selection. The simulation results indicate that the scheme is able to automatically adjust the impedance matching network according to the changes of the relative positions between Tx and Rx coils to achieve high energy transfer power and efficiency.
\end{abstract}

Keywords: multi-coil; wireless power transmission; coupled magnetic resonance; adaptive impedance matching

\section{Introduction}

With the prevalence of electronic devices in daily life, the limitations of wired energy transmission have become increasingly apparent. Wireless power transfer (WPT) has gained widespread popularity as it reduces reliance on wired connections. WPT can be divided into three main categories: magnetic induction [1], coupled magnetic resonance [2] and microwave radiation [3]. Among them, CMR offers unique advantages, as it has higher transfer distance and power transfer efficiency [4,5]. In [6], Karalis et al. demonstrated that CMR can achieve $40 \%$ wireless power transfer efficiency (PET) at a distance of $2 \mathrm{~m}$. Thus, CMR technology has a variety of potential applications, e.g., sensors, wearable mobile devices [7] and electronic vehicles [8].

However, energy distance and efficiency are still in need of further improvement. Single Tx and Rx CMR not only has low energy transfer distance and efficiency, but the application range is also limited. Recently, multiple-input multiple-output (MIMO) has been proposed. Based on the number of coils, CMR can be divided into single-input single-output (SISO), single-input multiple-output (SIMO), multiple-input single-output (MISO) and MIMO. Jadidian et al. proposed the idea of MIMO CMR systems, which attracted significant attention within the field [4]. Nguyen et al. investigated the effect of MIMO WPT system on the transmitting power [9], while Aoki et al. have calculated the maximum transmitting efficiency of MIMO WPT [10]. In addition Lang et al. proposed that MIMO can transfer higher power than SISO or SIMO [11]. Compared to the other 
CMR models, MIMO is able to carry multiple users at the same time while achieving high energy transfer power.

Impedance mismatch is the main problem for CMR. Input impedance of the system varies with the position between the Tx and Rx coils. The input impedance of the system directly affects the variation of the reflection coefficient and thus the efficiency and energy transfer power [12]. Therefore, the impedance mismatch in CMR is a problem worth focusing on. Researchers have begun looking for ways to solve the impedance mismatch problem.

Impedance mismatch is not a novel problem. To solve the problem of impedance mismatch, several solutions have been proposed based on impedance mismatching networks (IMNs). Classical IMNs are divided into L-type, $\pi$-type and T-type networks [6]. Liu et al. proposed a one-side automated discrete impedance matching network system [13]. Kim et al. propose a impedance matching network for the case of one transmitter coupled to multiple receivers [14]; however, its application scope is small. Lee et al. have proposed an impedance matching analysis method for CMR systems containing multiple repeaters [15], while $\mathrm{Li}$ et al. have used a BP neural network to realize impedance match [16]. Jeong et al. have used neural networks and IMN techniques to achieve coil selection that maximises power transfer efficiency [17]. Zhai et al. have achieved impedance matching by changing the operating frequency of the system. [18]. Kin et al. have achieved impedance matching by using automated source frequency tracking [19], but this method shows slow response. Lee et al. have used $\pi$-type impedance matching networks for wireless energy transfer [20]. Lu et al. have used printed spiral coils with impedance matching networks for wireless energy transfer [21]. Fujiki et al. have applied impedance matching networks to wireless energy transmission systems with repeaters for the purpose of reducing frequency splitting [22]. Boo et al. have proposed an analysis and impedance matching optimization of a wireless energy transfer system with a rotating coil [23]; however, it can only be used on SISO CMR systems.

Conventional SISO CMR systems are unable to fulfil high-powered energy transmission and flexible user-side combinations requirements. Impedance mismatch is more complex in MIMO. The circuit composition of MIMO is more flexible and the input impedance is affected by multiple coils, which are entangled together, making it complex to calculate the input impedance and analyse the IM for MIMO. IM in MIMO has yet to be fully investigated.

In this paper, we solve the problem of constructing a complex IM model for MIMO CMR system. The main contributes of this paper are as follows:

- We propose an analytical model which thoroughly addresses the complexity of the MIMO CMR system. The MIMO CMR system model can simulate and calculate the current and voltage values of the MIMO CMR system before and after impedance matching.

- $\quad$ Based on the MIMO CMR system model, we design an impedance matching network (IMN) and derive an optimal impedance matching (IM) solution.

- We also propose an adaptive impedance matching scheme for magnetic MIMO wireless power transfer system. The scheme, which is based on an analytical model of MIMO CMR system, achieves optimal values for transmission power and efficiency through IMN and coil selection. Our proposed scheme is able to automatically adjust the impedance matching network according to the change in relative position between the Tx and Rx coils to achieve high energy transfer power and efficiency. The simulation results indicate that a MIMO CMR system with IMN can achieve higher energy transfer power and efficiency than a MIMO CMR system without IMN.

In the rest of the paper, we analyze the MIMO CMR system in Section 2. Based on this, the problem simulation of power transfer efficiency and MIMO-IM is presented in Section 3. We design a IMN for MIMO CMR system and derive a theoretical approach for achieving MIMO CMR system IM in Section 4. We design an automatic control scheme 
for optimal values of transmission power and efficiency through IMN and coil selection in Section 5. The final simulation results are presented in Section 6.

\section{System Model}

The circuit diagram of a MIMO CMR system is shown in Figure 1. The power source provides AC voltage and the internal resistance is equivalent to $Z_{S}=R_{S}$. The Tx consists of a resonant capacitor $C_{T}$, a resistance $R_{T}$ and a Tx coil with inductance $L_{T}$. Similarly, the receiver consists of a Rx coil with a resonant capacitor $C_{R}$, the resistance $R_{R}$ and the inductance $L_{R}$ of the Rx coil. The input impedance varies with the mutual inductance $M$, which relates to the position and distance between the transmitting and receiving coils, and results in an impedance mismatch. At the same time, the addition of multiple coils results in coils coupling to each other, making the input impedance $Z_{\text {in }}$ analysis more complex.

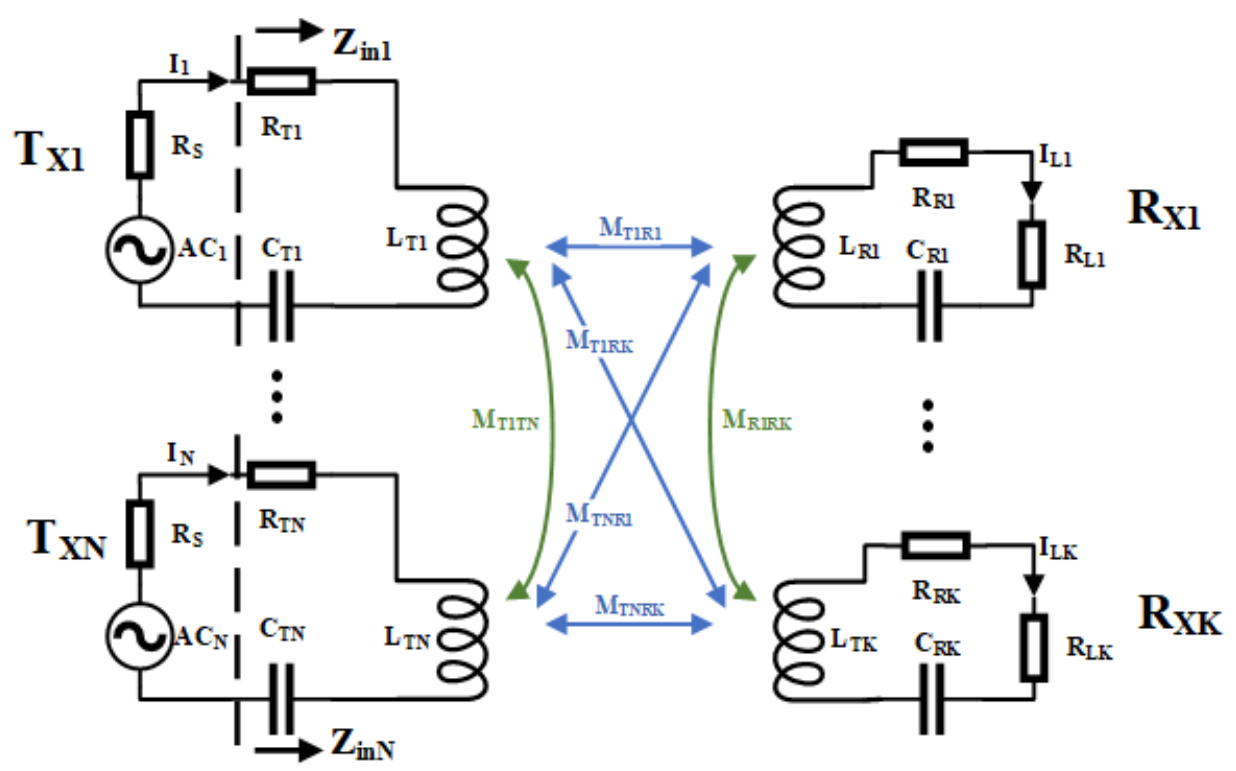

Figure 1. MIMO CMR system.

Assuming that the current went through the $N$ th transmitter coil is $I_{N}$ and the current went through the $K$ th receiver coil is $I_{L K}$. Since the system operates in resonance, based on Kirchhoff's voltage law (KVL), we have expressions of every circuit:

Tx:

$$
\left\{\begin{array}{c}
V_{1}=I_{1} R_{T 1}+I_{1} R_{S}+\sum_{i \neq 1}^{N} j \omega M_{T_{1} T_{i}} I_{i}- \\
\sum_{i=1}^{K} j \omega M_{T_{1} R_{i}} I_{L i} \\
\vdots \\
V_{N}=I_{N} R_{T N}+I_{N} R_{S}+\sum_{i \neq N}^{N} j \omega M_{T_{N} T_{i}} I_{i}- \\
\sum_{i=1}^{K} j \omega M_{T_{N} R_{i}} I_{L i}
\end{array}\right.
$$

Rx:

$$
\left\{\begin{array}{c}
\sum_{i=1}^{N} j \omega M_{T_{i} R_{1}} I_{i}-\sum_{i \neq 1}^{K} j \omega M_{R_{i} R_{1}} I_{L i}=I_{L 1}\left(R_{L 1}+R_{R 1}\right) \\
\vdots \\
\sum_{i=1}^{N} j \omega M_{T_{i} R_{K}} I_{i}-\sum_{i \neq K}^{K} j \omega M_{R_{i} R_{K}} I_{L i}=I_{L K}\left(R_{L K}+R_{R K}\right)
\end{array}\right.
$$

where $M$ was mutual inductance, $V$ was voltage of source and $I$ was current. Equation (1) is expression of Tx circuits and Equation (2) is expression of Rx circuits. By changing Equations (1) and (2) into matrix form, we can obtain Equations (3) and (4). 
Tx:

$$
\begin{array}{r}
\mathbf{V}=\left[\begin{array}{cccc}
R_{T 1}+R_{S} & j \omega M_{T_{1} T_{2}} & \cdots & j \omega M_{T_{1} T_{N}} \\
j \omega M_{T_{2} T_{1}} & R_{T 2}+R_{S} & \cdots & j \omega M_{T_{2} T_{N}} \\
\vdots & \vdots & \ddots & \vdots \\
j \omega M_{T_{N} T_{1}} & j \omega M_{T_{N} T_{2}} & \cdots & R_{T N}+R_{S}
\end{array}\right] \\
\times \mathbf{I}-\left[\begin{array}{cccc}
j \omega M_{T_{1} R_{1}} & \cdots & j \omega M_{T_{1} R_{K}} \\
j \omega M_{T_{2} R_{1}} & \cdots & j \omega M_{T_{2} R_{K}} \\
\vdots & \ddots & \\
j \omega M_{T_{N} R_{1}} & \cdots & j \omega M_{T_{N} R_{K}}
\end{array}\right] \times \mathbf{I}_{\mathbf{L}}
\end{array}
$$

Rx:

$$
\begin{gathered}
{\left[\begin{array}{cccc}
j \omega M_{T_{1} R_{1}} & j \omega M_{T_{2} R_{1}} & \cdots & j \omega M_{T_{N} R_{1}} \\
\vdots & \vdots & \ddots & \vdots \\
j \omega M_{T_{1} R_{K}} & j \omega M_{T_{2} R_{K}} & \cdots & j \omega M_{T_{N} R_{K}}
\end{array}\right] \times \mathbf{I}} \\
=\left[\begin{array}{ccc}
\left(R_{L 1}+R_{R 1}\right) & \cdots & j \omega M_{R_{K} R_{1}} \\
\vdots & \ddots & \vdots \\
j \omega M_{R_{1} R_{K}} & \cdots & \left(R_{L K}+R_{R K}\right)
\end{array}\right] \times \mathbf{I}_{\mathbf{L}}
\end{gathered}
$$

where Tx voltage vector was $\mathbf{V}=\left[\begin{array}{llll}V_{1} & V_{2} & \cdots & V_{N}\end{array}\right]^{T}$, Tx current vector was $\mathbf{I}=\left[\begin{array}{llll}I_{1} & I_{2} & \cdots & I_{N}\end{array}\right]^{T}$ and Rx current vector was $\mathbf{I}_{\mathbf{L}}=\left[\begin{array}{llll}I_{L 1} & I_{L 2} & \cdots & I_{L K}\end{array}\right]^{T}$. Substituting Equation (4) into Equation (3) and simplifying it we get Equation (5)

$$
\mathbf{I}=\left(\mathbf{T}_{\mathbf{z 1}}-\mathbf{T}_{\mathbf{z 2}} \mathbf{T}_{\mathbf{z 3}}{ }^{-1} \mathbf{T}_{\mathbf{z 4}}\right)^{-1} \mathbf{V}
$$

where matrix $\mathbf{T}_{\mathbf{z 1}}, \mathbf{T}_{\mathbf{z} 2}, \mathbf{T}_{\mathbf{z} 3}$ and $\mathbf{T}_{\mathbf{z} 4}$ are the matrices used to simplify the operation, which include all information of the system as showed below.

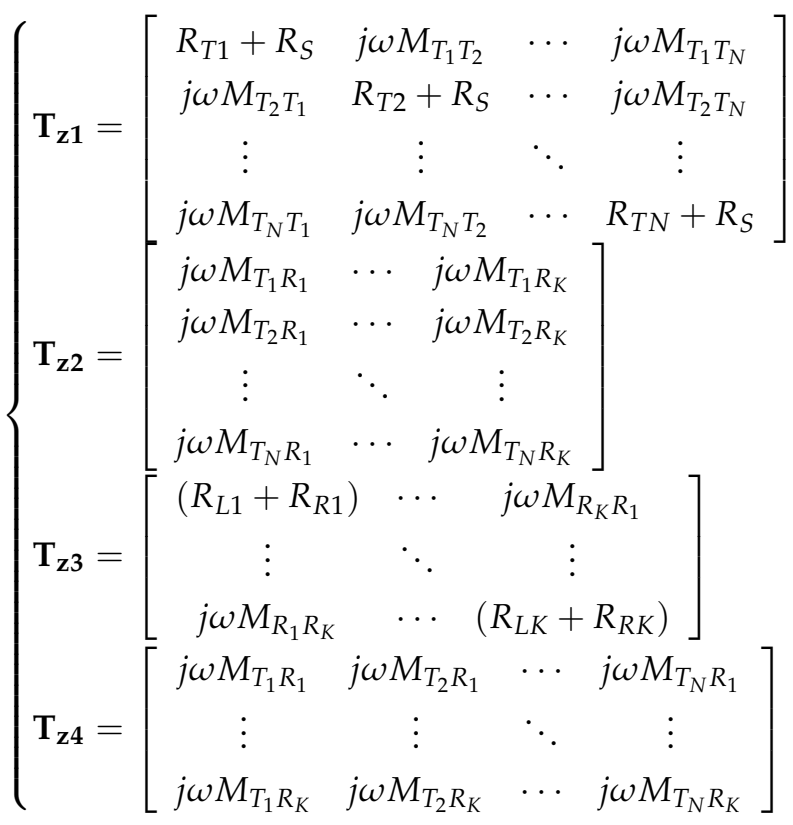


The total impedance can be obtained by dividing the supply voltage $V$ by the current $I$ obtained from Equation (5). The total impedance minus the internal resistance of the supply $R_{S}$ can obtain the input impedance $Z_{i n}$. The expression of input impedance $Z_{i n}$ is shown in Equation (7).

$$
Z_{\text {in }}=\frac{V}{I}-R_{S}
$$

where voltage $V$ and current $I$ follows Equation (5) according by the mutual inductance $M$. Therefore, when the coil position is changed, the mutual inductance $M$ changes, which in turn changes the input impedance $Z_{i n}$.

\section{Problem Formulation}

\subsection{Power Transfer Efficiency}

Power transfer efficiency of the CMR system $\eta_{s y s}$ is very important. Figure 2 shows the equivalent circuit diagram of the CMR system. The energy from the AC source is transferred to the load resistor through the mutual inductance $M_{T R}$ between the Tx coil and the Rx coil. The power from AC source $P_{S}$, the input power $P_{\text {in }}$, the output power $P_{\text {out }}$ and the load received power $P_{\text {load }}$ are shown in Figure 2 . The power transfer efficiency is obtained from Equation (8).

$$
\eta_{\text {sys }}=\eta_{\text {match }} \eta_{\text {trans }} \eta_{\text {rec }}=\frac{P_{\text {in }}}{P_{S}} \times \frac{P_{\text {out }}}{P_{\text {in }}} \times \frac{P_{\text {load }}}{P_{\text {out }}}=\frac{P_{\text {load }}}{P_{S}}
$$

where $\eta_{\text {match }}, \eta_{\text {trans }}$ and $\eta_{\text {rec }}$ are the matching system efficiency, transmission system efficiency and receiving efficiency, respectively. The expression of matching system efficiency $\eta_{\text {match }}$ is illustrated in Equation (9).

$$
\eta_{\text {match }}=1-\left|S_{11}\right|^{2}=\frac{4 Z_{\text {in }} R_{S}}{\left(Z_{\text {in }}+R_{S}\right)^{2}}=\left(1-\frac{I R_{s}}{V}\right) \frac{4 I R_{S}}{V}
$$

where $S_{11}$ is the $S$ parameter of the matching network; $Z_{i n}$ is the input impedance; $V$ is the Tx voltage; $I$ is the Tx current; $R_{S}$ is the internal resistance of the power supply. From Equation (9), when $Z_{i n}=Z_{S}$, the optimal value of $S_{11}=0$, and the maximum value of $\eta_{\text {match }}$ can be obtained the matching system efficiency $\eta_{\text {match }}=1$. When $Z_{\text {in }} \neq R_{S}, S_{11} \neq 0$, the matching system efficiency $\eta_{\text {match }}$ is not optimal, $\eta_{\text {match }}<1$, and the power transfer efficiency of the WPT system $\eta_{s y s}$ cannot reach the maximum value. Thus, the major problem of our paper is to adjust $Z_{i n}$ to achieve maximum $\eta_{\text {sys }}$.

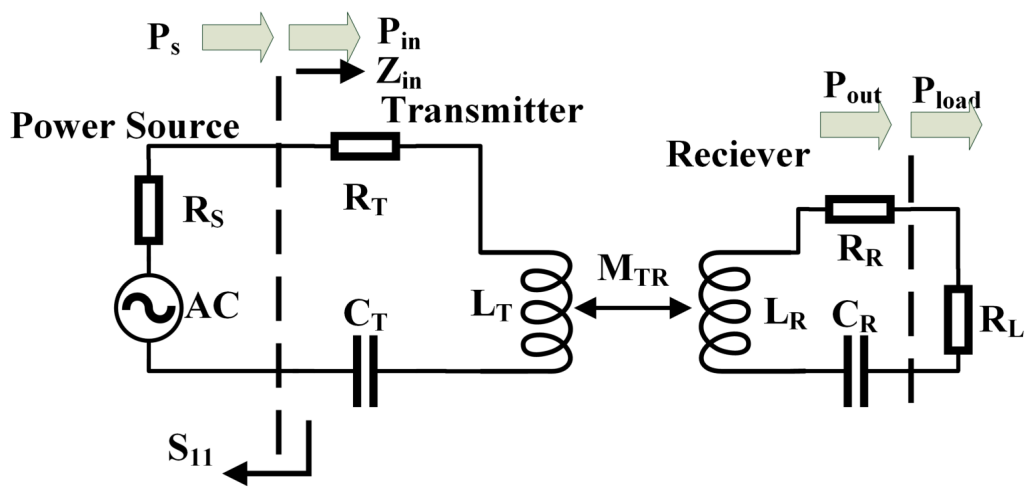

Figure 2. Equivalent circuit diagram of the CMR system.

\subsection{Impedance Matching}

To solve impedance mismatching problem, the IMN is used. Compared with other types of networks, L-type network is simple in structure and have a considerable range of variation. However, L-type IMN cannot be used in high-frequency areas. This disadvantage is neglected because the MIMO CMR system model we constructed is not in the high- 
frequency area. We apply L-type IMN in the MIMO CMR system. The L-type impedance matching network can be subdivided into two types: the L-type IMN and the reverse L-type IMN, which is demonstrated in Figure 3. Because there are branches in the L-type IMN circuit, and the branches will make the coil current different from the power source current, it is quite complicated to analyze the energy transfer after adding IMN. The L-type IMN and the reverse L-type IMN are used in different situations. L-type IMN is used when Tx coil closed to Rx coil. Reverse L-type IMN is userd frequently when Tx coil stay away from Rx coil.
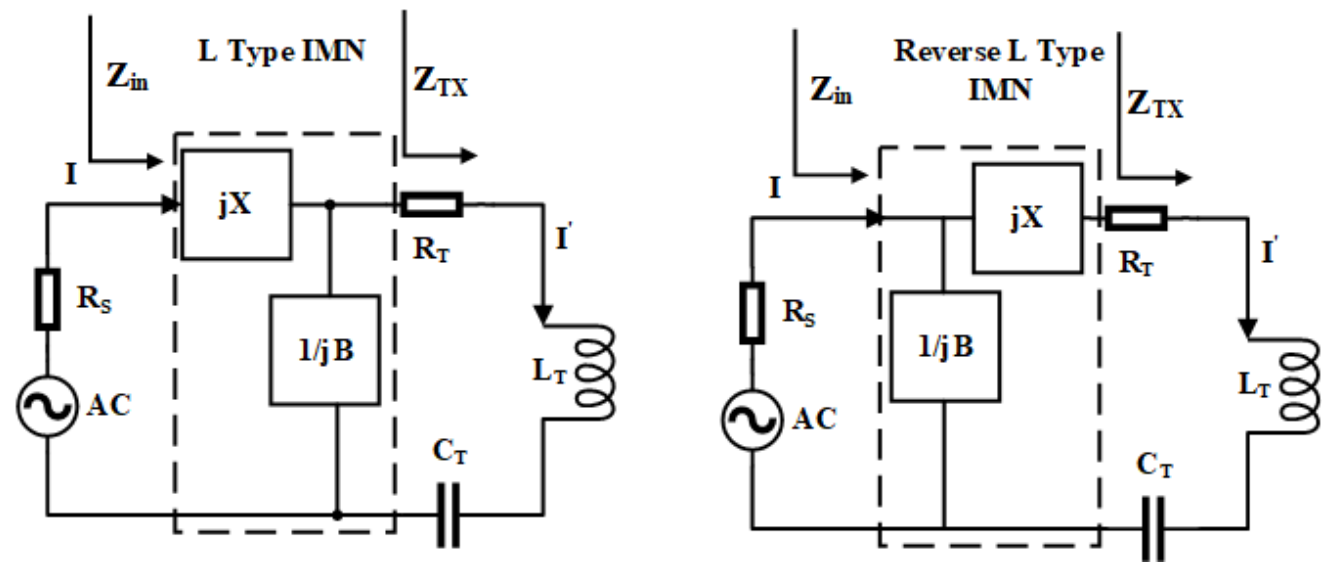

Figure 3. L-type IMN and reverse L-type IMN.

When $\Re\left(Z_{T x}\right)>R_{S}$, an L-type IMN is used, and in order to achieve impedance matching $\left(Z_{i n}=R_{S}\right)$, the component parameters in the IMN should satisfy Equation (10).

$$
R_{S}=\frac{1}{j B+\frac{1}{Z_{T X}}}+j X
$$

where $Z_{T X}$ represents the equivalent impedance on the right side of the IMN; $R_{T x}$ and $X_{T x}$ represent the real and imaginary parts of $Z_{T X}$. By separating the real and imaginary parts of Equation (10), we obtain the component parameters $X$ and $B$ of the L-type IMN, where $B$ is included in $X$.

$$
\begin{gathered}
X=\frac{1}{B}+\frac{R_{S} X_{T X}}{R_{T X}}-\frac{R_{S}}{B R_{T X}} \\
B=\frac{X_{T X} \pm \sqrt{R_{T X} / R_{S}} \sqrt{R_{T X}^{2}+X_{T X}^{2}-R_{S} R_{T X}}}{R_{T X}^{2}+X_{T X}^{2}}
\end{gathered}
$$

Conversely, when $\Re\left(Z_{T x}\right)<R_{S}$, a reverse L-type IMN is a better choice, and the component parameters in the IMN are, thus, varied and satisfy Equation (13).

$$
\frac{1}{R_{S}}=\frac{1}{j X+R_{T X}+j X_{T X}}+j B
$$

By separating the real and imaginary parts of Equation (13), we obtain the component parameters $X$ and $B$ of the reverse L-type IMN.

$$
\begin{gathered}
X= \pm \sqrt{R_{T X}\left(R_{S}-R_{L}\right)}-X_{T X} \\
B= \pm \frac{\sqrt{\left(R_{S}-R_{T X}\right) / R_{T X}}}{R_{S}}
\end{gathered}
$$


From Equation (10) to Equation (15), it can be seen that the component parameters $X$ and $B$ of IMN are influenced by the equivalent impedance $Z_{T x}$ on the right side of IMN, and when $Z_{T x}$ changes, $X$ and $B$ change as well.

\subsection{MIMO System Impedance Matching Network}

From Equation (10) to Equation (15), it can be seen that the values of component parameters $X$ and $B$ are only affected by their respective equivalent impedance $Z_{T x}$ and not by the other coils. Therefore, the IMN of each transmitter can be analyzed independently. The analysis and calculation of impedance matching networks are relatively independent. Therefore, the IMN of each Tx coil behaves as two types, each corresponding to the Ltype impedance matching network and the reverse L-type impedance matching network proposed in the previous section.

To reduce the space of the IMN, we use the IMN model shown in Figure 4, which consists of two inductance matrices and one capacitance matrix. As shown in Figure 4, by controlling single-pole multiple-throw switches, the inductance matrix and capacitance matrix are combined with each other to be able to form different impedance matching networks. By pre-simulatuon shown in Section 5, at a coil distance of $0.001 \mathrm{~m}$ to $0.5 \mathrm{~m}$, the component of capacitor matrix $B$ range from $40 \mathrm{pF}$ to $15 \mathrm{nF}$, the component of inductor matrix $X_{L}$ range from $270 \mathrm{nH}$ to $12 \mu \mathrm{H}$, and the component of inductor matrix $X_{R L}$ range from $29 \mathrm{nH}$ to $550 \mathrm{nH}$. All of the components are cheap and easy to get. From the application point of view, the use of simple and inexpensive components to achieve optimal transmission efficient and transmission energy is feasible and effective. In order to achieve impedance matching, the component parameters of the IMN should satisfy Equation (16).

$$
R_{S}=j X_{L}+\frac{1}{j B+\frac{1}{j X_{R L}+Z_{T X}}}
$$

Equation (16) shows that at $\Re\left(Z_{T x}\right)>R_{S}$, the IMN behaves as a L-type IMN by shorting $X_{R L}$, i.e., $X_{R L}=0$. The IMN component parameters are expressed in Equation (17).

$$
\left\{\begin{array}{l}
X_{L}=\frac{1}{B}+\frac{R_{S} X_{T X}}{R_{T X}}-\frac{R_{S}}{B R_{T X}} \\
X_{R L}=0 \\
B=\frac{X_{T X} \pm \sqrt{R_{T X} / R_{S}} \sqrt{R_{T X}^{2}+X_{T X}^{2}-R_{S} R_{T X}}}{R_{T X}^{2}+X_{T X}^{2}}
\end{array}\right.
$$

Similarly, when $\Re\left(Z_{T x}\right)<R_{S}$, the IMN behaves as a reverse L-type IMN by shorting $X_{L}$, i.e., $X_{L}=0$. The IMN component parameters are expressed in Equation (18).

$$
\left\{\begin{array}{l}
X_{L}=0 \\
X_{R L}= \pm \sqrt{R_{T X}\left(R_{S}-R_{L}\right)}-X_{T X} \\
B= \pm \frac{\sqrt{\left(R_{S}-R_{T X}\right) / R_{T X}}}{R_{S}}
\end{array}\right.
$$

As shown in Equations (17) and (18), we can obtain the L-type IMN by shorting $X_{R L}$. Similarly, we can obtain the reverse L-type IMN by shorting $X_{L}$. In order to analyze the effect of IMN on the power transfer efficiency of the MIMO CMR system. We choose the IMN component parameters $X_{L}, X_{R L}$ and $B$ to maximize the transfer efficiency $\eta_{s y s}$.

$$
\begin{aligned}
& \left(X_{L}, X_{R L}, B\right)=\arg \max \eta_{\text {sys }} \\
& \text { s.t. } \Re\left(Z_{T x}\right)>0 .
\end{aligned}
$$


where $\Re\left(Z_{T x}\right)>0$ means that the Tx coils must only transfer energy. If the appropriate, $X_{L}, X_{R L}$ and $B$ are selected according to Equations (17) and (18), input impedance $Z_{\text {in }}=R_{S}$ can be obtained, and thus the power transfer efficiency of the WPT system $\eta_{s y s}$ reaches the highest value. Therefore, we only need to analyze the calculation of the impedance matching network to obtain the general equation for the L-type IMN and the reverse L-type IMN.

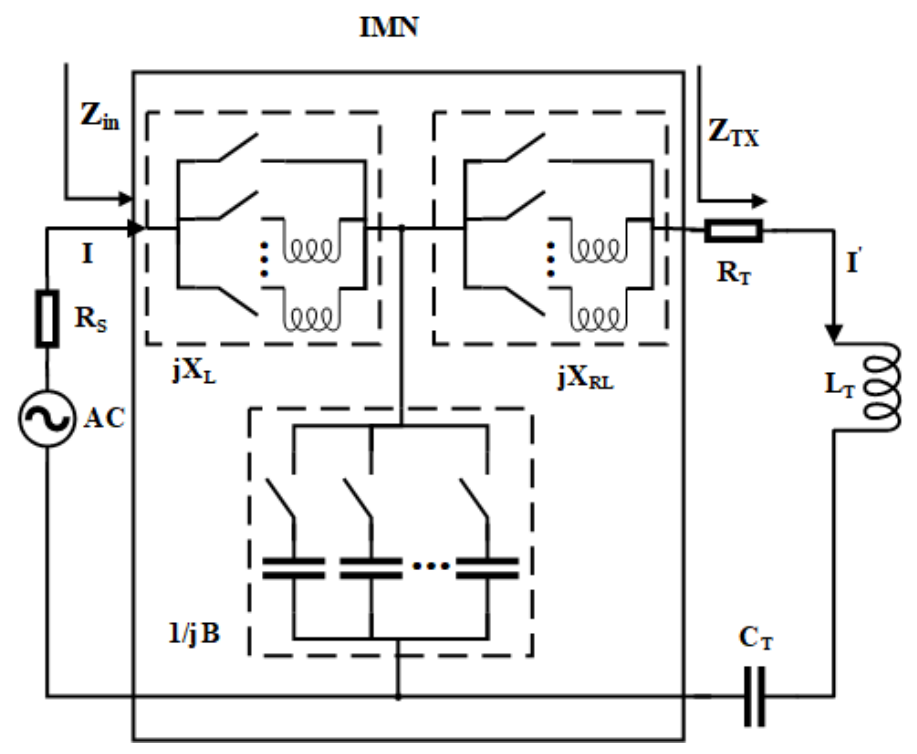

Figure 4. Pseudo-T-type impedance matching network.

\section{Optimal MIMO Impedance Matching Solution}

The coupling of multiple coils can make the calculation of impedance matching very complex. A brief circuit diagram of MIMO CMR system impedance matching is shown in Figure 5. From the diagram we can see that each transmitting coil is equipped with an impedance matching network, and each impedance matching network is analyzed and calculated independently of the other impedance matching networks. The power source $I$ and the transmitter coil current $I^{\prime}$ are shown in Figure 5.

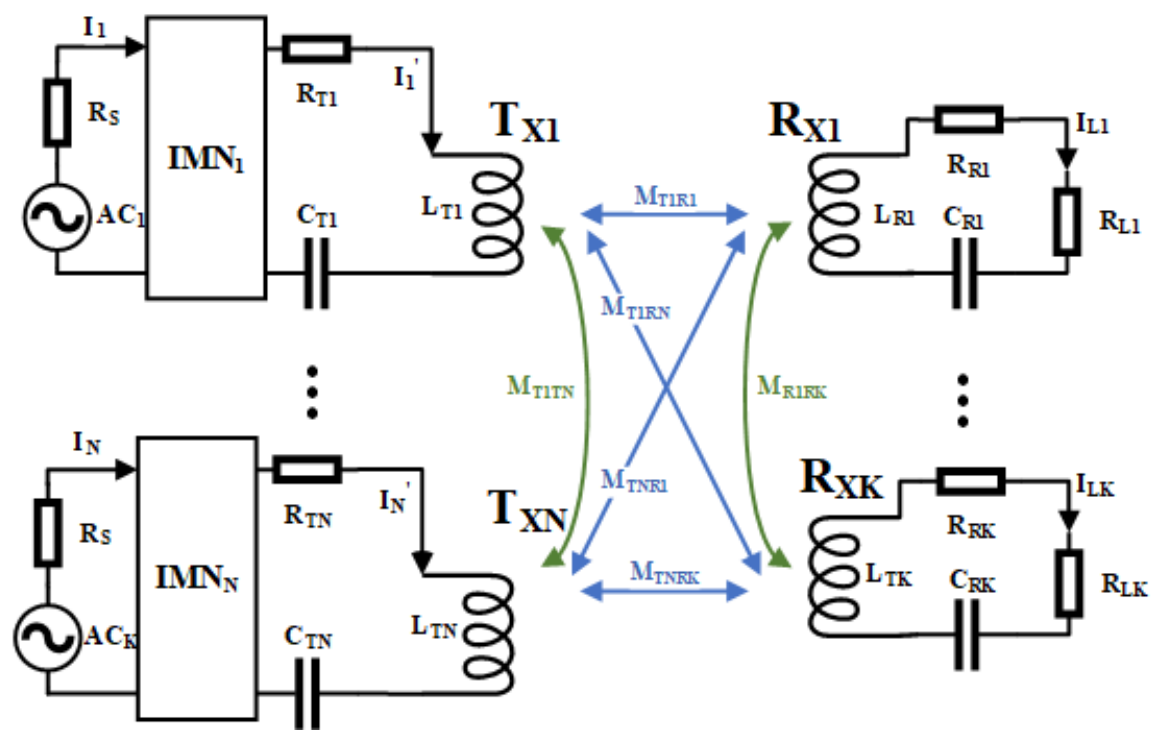

Figure 5. Impedance matching networks for MIMO CMR system. 
The impedance matching of the MIMO system can be achieved by using the IMN of Figure 4 in the MIMO system of Figure 5. Take the $l$ th Tx for instance, where $l \in\{1 \cdots N\}$. Based on KVL, the expression of the $l$ th Tx is shown in Equation (19).

The $l$ th Tx:

$$
\left\{\begin{array}{l}
V_{l}=I_{l} R_{S}+j X_{L l} I_{l}+\frac{I_{l}}{j B_{l}}-\frac{I_{l}^{\prime}}{j B_{l}} \\
\frac{I_{l}}{j B_{l}}=\frac{I_{l}^{\prime}}{j B_{l}}+I_{l}^{\prime} R_{T l}+j X_{R L l} I_{1}^{\prime}+\sum_{i \neq l}^{N} j \omega M_{T_{l} T_{i}} I_{i}^{\prime}-\sum_{i=1}^{K} j \omega M_{T_{l} R_{i}} I_{L i}
\end{array}\right.
$$

where $V_{l}$ is the voltage of the voltage source; $I_{l}$ is the current flowing through the voltage source; $I_{l}^{\prime}$ is the Tx coil current; $X_{L} l, X_{R L l}$, and $B_{l}$ are all component parameters of IMN; $M_{T R}$ is the mutual inductance between Tx and Rx; $M_{T T}$ is the mutual inductance between Tx and Tx; $R_{S}$ is the internal resistance of the voltage source; and $R_{T}$ is the resistance of the Tx coil.

Take the $p$ th Rx for instance, where $p \in\{1 \cdots K\}$. Based on KVL, the expression of current and voltage relationship for the $p$ th $\mathrm{Rx}$ is shown in Equation (19). It shows that Tx coil is influenced by other Tx coils and Rx coils.

The $p$ th Rx:

$$
\sum_{i=1}^{N} j \omega M_{T_{i} R_{p}} I_{i}^{\prime}-\sum_{i \neq p}^{K} j \omega M_{R_{p} R_{i}} I_{L i}=I_{L p}\left(R_{L p}+R_{R p}\right)
$$

where $I_{L p}$ is the current flowing through the Rx coil; $I_{p}^{\prime}$ is the current flowing through the Tx coil; $M_{T R}$ is the mutual inductance between Tx and Rx; $M_{R R}$ is the mutual inductance between Rx and Rx; $R_{L p}$ is the load resistance; and $R_{R p}$ is the resistance of the Rx coil. Equation (20) present that Rx coil is influenced by other Tx coils and Rx coils.

By changing Equations (19) and (20) into matrix form, we can obtain Equations (21) and (22).

$$
\begin{aligned}
& \mathbf{T x}: \mathbf{I}^{\prime}=\left[\begin{array}{cccc}
G_{1} & 0 & \cdots & 0 \\
0 & G_{2} & \cdots & 0 \\
\vdots & \vdots & \ddots & \vdots \\
0 & 0 & \cdots & G_{N}
\end{array}\right] \mathbf{I}-\left[\begin{array}{cccc}
j B_{1} & 0 & \cdots & 0 \\
0 & j B_{2} & \cdots & 0 \\
\vdots & \vdots & \ddots & \vdots \\
0 & 0 & \cdots & j B_{N}
\end{array}\right] \mathbf{V} \\
& \mathbf{R x}:\left[\begin{array}{c}
\mathbf{I} \\
\mathbf{I}_{\mathbf{L}}
\end{array}\right]=\left[\begin{array}{ccccccc}
D_{1} & -S_{1} M_{T_{1} T_{2}} & \cdots & -S_{1} M_{T_{1} T_{N}} & S_{1} M_{T_{1} R_{1}} & \cdots & S_{1} M_{T_{1} R_{K}} \\
-S_{2} M_{T_{2} T_{1}} & D_{2} & \cdots & -S_{2} M_{T_{2} T_{N}} & S_{2} M_{T_{2} R_{1}} & \cdots & S_{2} M_{T_{2} R_{K}} \\
\vdots & \vdots & \cdots & \vdots & \vdots & \cdots & \vdots \\
-S_{N} M_{T_{N} T_{2}} & -S_{N} M_{T_{N} T_{2}} & \cdots & D_{N} & S_{N} M_{T_{N} R_{1}} & \cdots & S_{N} M_{T_{N} R_{K}} \\
F_{1} M_{T_{1} R_{1}} & F_{1} M_{T_{2} R_{1}} & \cdots & F_{1} M_{T_{N} R_{1}} & 0 & \cdots & -F_{1} M_{R_{1} R_{K}} \\
\vdots & \vdots & \ddots & \vdots & \vdots & \ddots & \vdots \\
F_{K} M_{T_{1} R_{K}} & F_{K} M_{T_{2} R_{K}} & \cdots & F_{K} M_{T_{N} R_{K}} & -F_{K} M_{R_{K} R_{1}} & \cdots & 0
\end{array}\right] \times\left[\begin{array}{c}
\mathbf{I}^{\prime} \\
\mathbf{I}_{\mathbf{L}}
\end{array}\right]
\end{aligned}
$$

where Tx current vector $\mathbf{I}^{\prime}=\left[\begin{array}{llll}I_{1}^{\prime} & I_{2}^{\prime} & \cdots & I_{N}^{\prime}\end{array}\right]^{T}$, the voltage source current vector $\mathbf{I}=\left[\begin{array}{llll}I_{1} & I_{2} & \cdots & I_{N}\end{array}\right]^{T}$, Tx voltage vector $\mathbf{V}=\left[\begin{array}{llll}V_{1} & V_{2} & \cdots & V_{N}\end{array}\right]^{T}$ and Rx current 
vector $\mathbf{I}_{\mathbf{L}}=\left[\begin{array}{llll}I_{L 1} & I_{L 2} & \cdots & I_{L K}\end{array}\right]^{T}$. Parameters $G, D, S$ and $F$ are used to simplify the operation, which is expressed in Equation (23).

$$
\left\{\begin{array}{l}
G_{i}=j B_{i} R_{S}-B_{i} X_{L i}+1 \\
D_{i}=1-B_{i} X_{R L i}+j B_{\mathrm{i}} R_{\mathrm{Ti}} \\
S_{i}=B_{i} \omega \\
F_{i}=j \omega /\left(R_{L 1}+R_{R 1}\right)
\end{array}\right.
$$

Substituting Equation (22) into Equation (21) and simplifying it, we get Rx current vector in Equation (24) and the current vector went through the voltage source in Equation (25)

$$
\begin{aligned}
& \mathbf{I}_{\mathbf{L}}=\mathbf{T}_{\mathbf{1}}{ }^{-1} \mathbf{T}_{\mathbf{2}}\left(\mathbf{T}_{\mathbf{3}}+\mathbf{T}_{\mathbf{4}} \mathbf{T}_{\mathbf{1}}{ }^{-1} \mathbf{T}_{\mathbf{2}}\right)^{-1} \mathbf{T}_{\mathbf{5}} \mathbf{V}=\mathbf{Y}_{\mathbf{1}} \mathbf{V} \\
& \mathbf{I}=\mathbf{T}_{\mathbf{6}}^{-\mathbf{1}}\left[\left(\mathbf{T}_{\mathbf{3}}+\mathbf{T}_{\mathbf{4}} \mathbf{T}_{\mathbf{1}}{ }^{-1} \mathbf{T}_{\mathbf{2}}\right)^{-1}+\mathbf{E}\right] \mathbf{T}_{\mathbf{5}} \mathbf{V}=\mathbf{Y}_{\mathbf{2}} \mathbf{V}
\end{aligned}
$$

where each current vector is related to the voltage vector by expressions: $\mathbf{Y}_{\mathbf{1}}=\mathbf{T}_{\mathbf{1}}{ }^{-1} \mathbf{T}_{\mathbf{2}}\left(\mathbf{T}_{3}+\right.$ $\left.\mathbf{T}_{4} \mathbf{T}_{1}{ }^{-1} \mathbf{T}_{2}\right)^{-1} \mathbf{T}_{5}, \mathbf{Y}_{2}=\mathbf{T}_{6}^{-1}\left[\left(\mathbf{T}_{3}+\mathbf{T}_{4} \mathbf{T}_{1}^{-1} \mathbf{T}_{2}\right)^{-1}+\mathbf{E}\right] \mathbf{T}_{5}$. Matrix $\mathbf{T}_{1}, \mathbf{T}_{2}, \mathbf{T}_{3}, \mathbf{T}_{4}, \mathbf{T}_{5}$ and $\mathbf{T}_{6}$ are used to simplify the operation, which showed in Equation (26). $\mathbf{E}$ is the expression of identity matrix.

$$
\begin{aligned}
& \mathbf{T}_{\mathbf{1}}=\left[\begin{array}{ccc}
1 & \cdots & F_{1} M_{R_{1} R_{K}} \\
\vdots & \ddots & \vdots \\
F_{K} M_{R_{K} R_{1}} & \cdots & 1
\end{array}\right] \\
& \mathbf{T}_{\mathbf{2}}=\left[\begin{array}{cccc}
F_{1} M_{T_{1} R_{1}} & F_{1} M_{T_{2} R_{1}} & \cdots & F_{1} M_{T_{N} R_{1}} \\
\vdots & \vdots & \ddots & \vdots \\
F_{K} M_{T_{1} R_{K}} & F_{K} M_{T_{2} R_{K}} & \cdots & F_{K} M_{T_{N} R_{K}}
\end{array}\right] \\
& \mathbf{T}_{3}=\left[\begin{array}{cccc}
D_{1} G_{1}-1 & -S_{1} M_{T_{1} T_{2}} G_{1} & \cdots & -S_{1} M_{T_{1} T_{N}} G_{1} \\
-S_{2} M_{T_{2} T_{1}} G_{2} & D_{2} G_{2}-1 & \cdots & -S_{2} M_{T_{2} T_{N}} G_{2} \\
\vdots & \vdots & \ddots & \vdots \\
-S_{N} M_{T_{N} T_{1}} G_{N} & -S_{N} M_{T_{N} T_{2}} G_{N} & \cdots & D_{N} G_{N}-1
\end{array}\right] \\
& \mathbf{T}_{4}=\left[\begin{array}{ccc}
\left(S_{1} M_{T_{1} R_{1}}\right) G_{1} & \cdots & \left.S_{1} M_{T_{1} R_{K}}\right) G_{1} \\
\left(S_{2} M_{T_{2} R_{1}}\right) G_{2} & \cdots & \left(S_{2} M_{T_{2} R_{K}}\right) G_{2} \\
\vdots & \ddots & \vdots \\
\left(S_{N} M_{T_{N} R_{1}}\right) G_{N} & \cdots & \left(S_{N} M_{T_{N} R_{K}}\right) G_{N}
\end{array}\right] \\
& \mathbf{T}_{\mathbf{5}}=\operatorname{diag}\left\{\left[\begin{array}{llll}
j B_{1} & j B_{2} & \cdots & j B_{N}
\end{array}\right]^{T}\right\} \\
& \mathbf{T}_{\mathbf{6}}=\operatorname{diag}\left\{\left[\begin{array}{llll}
G_{1} & G_{2} & \cdots & G_{N}
\end{array}\right]^{T}\right\}
\end{aligned}
$$


The load received power and power transfer efficiency can be obtained from the relationship between the load current and the voltage of the voltage source shown in Equation (24). The load received power is shown in Equation (27).

$$
\begin{aligned}
& \mathbf{P}_{\text {load }}=\mathbf{I}_{\mathbf{L}} \circ \overline{\mathbf{I}}_{\mathbf{L}}^{T} \operatorname{diag}\left\{\left[\begin{array}{llll}
R_{L 1} & R_{L 2} & \cdots & R_{L K}
\end{array}\right]^{T}\right\} \\
& =\mathbf{Y}_{\mathbf{1}} \mathbf{V} \circ{\overline{\left(\mathbf{Y}_{\mathbf{1}} \mathbf{V}\right)}}^{T} \operatorname{diag}\left\{\left[\begin{array}{llll}
R_{L 1} & R_{L 2} & \cdots & R_{L K}
\end{array}\right]^{T}\right\}
\end{aligned}
$$

where the load received power vector $\mathbf{P}_{\text {load }}=\left[\begin{array}{llll}P_{\text {load }}^{1} & P_{\text {load }}^{2} & \cdots & P_{\text {load }}^{K}\end{array}\right]^{T} \cdot \overline{\mathbf{I}}_{\mathbf{L}}$ is the Hermite matrix of $\mathbf{I}_{\mathbf{L}} \cdot R_{L}$ is the load resistance of $\mathrm{Rx}$. $\circ$ represent Hadamard product.

Similarly, according to the expression of the relationship between current and voltage in Equation (25), the power supply power can be obtained, as shown in Equation (28).

$$
\begin{array}{r}
\mathbf{P}_{\mathbf{S}}=\mathbf{I} \circ \overline{\mathbf{I}}^{T} \operatorname{diag}\left\{\left[\begin{array}{llll}
Z_{i n 1} & Z_{i n 2} & \cdots & Z_{i n N}
\end{array}\right]^{T}\right\} \\
=\mathbf{Y}_{\mathbf{2}} \mathbf{V} \circ{\overline{\left(\mathbf{Y}_{\mathbf{2}} \mathbf{V}\right)}}^{T} \operatorname{diag}\left\{\left[\operatorname{diag}\left(\mathbf{Y}_{\mathbf{2}} \mathbf{V}\right)\right]^{-1} \mathbf{V}-\left[\begin{array}{llll}
R_{S}^{1} & R_{S}^{2} & \cdots & R_{S}^{N}
\end{array}\right]^{T}\right\}
\end{array}
$$

where the load received power vector $\mathbf{P}_{\mathbf{S}}=\left[\begin{array}{llll}P_{S}^{1} & P_{S}^{2} & \cdots & P_{S}^{N}\end{array}\right]^{T} ; \overline{\mathbf{I}}$ is the Hermite matrix of $\mathbf{I} . Z_{i n}$ is the input impedance of MIMO CMR system. $R_{S}$ is the internal resistance of the power supply.

Combining Equations (27) and (28), the power transfer efficiency of the WPT system is shown in Equation (29).

$$
\eta_{\text {sys }}=\frac{\left\|\mathbf{P}_{\text {load }}\right\|_{1}}{\left\|\mathbf{P}_{\mathbf{S}}\right\|_{1}}
$$

The power transfer efficiency of a WPT system is expressed as the ratio of the total load received power to the total power output of the AC source. The total load received power and the total power output were influenced by IMN component parameters $X_{L}$, $X_{R L}$ and $B$. When impedance matching, i.e., $Z_{i n}=R_{S}$, IMN component parameters $X_{L}$, $X_{R L}$ and $B$ is obtained. Therefore, the optimal impedance matching solution is obtained. The power transfer efficiency of the WPT system $\eta_{\text {sys }}$ reaches its maximum value.

\section{Procedure of Adaptive Impedance Matching Scheme}

The adaptive impedance matching scheme can be visually displayed by Figure 6. By cycling the scheme in every $T$ seconds, real-time impedance matching can be achieved. Based on the Tx coil current vector and Tx coil voltage vector, the input impedance can be obtained. If the real part of the input impedance is less than zero, then this transmit coil is disconnected. Based on the real part of the input impedance, values of the impedance matching network are calculated. Micro-controller controls single-pole multiple-throw (SPMT) switchs to construct each IMN. Therefore, MIMO CMR system can achieve IM in the period of $T$ seconds.

Algorithms can be used for the simulation of MIMO real-time impedance matching. The algorithm is part of the scheme, and the all scheme is divided into four parts: measuring the coupling inductance $M$ between the calculation coils, Tx impedance measurement, selection of transmitter, IMN type decision and respective component values. The Algorithm 1 shows the detail. 


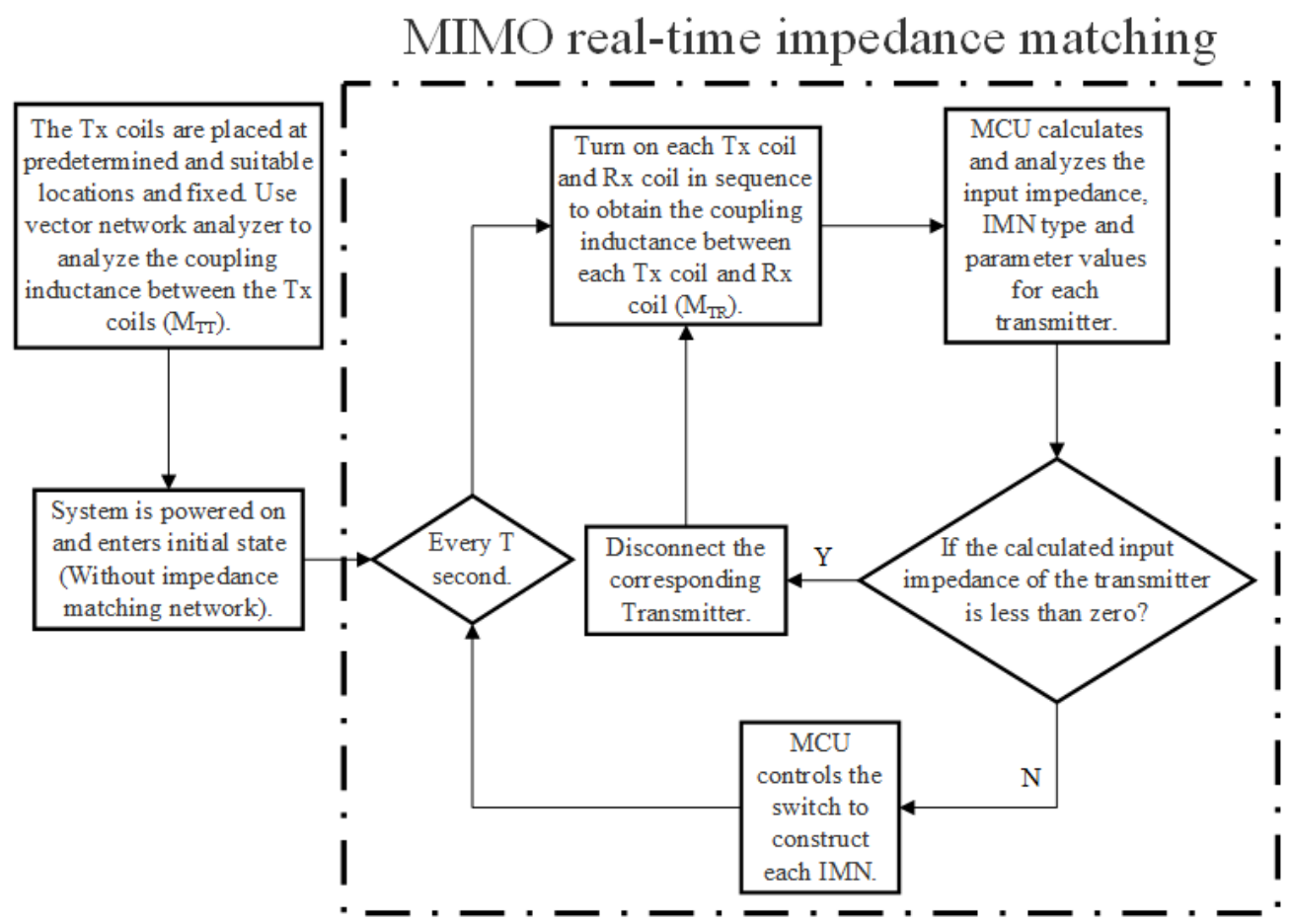

Figure 6. Adaptive impedance matching scheme.

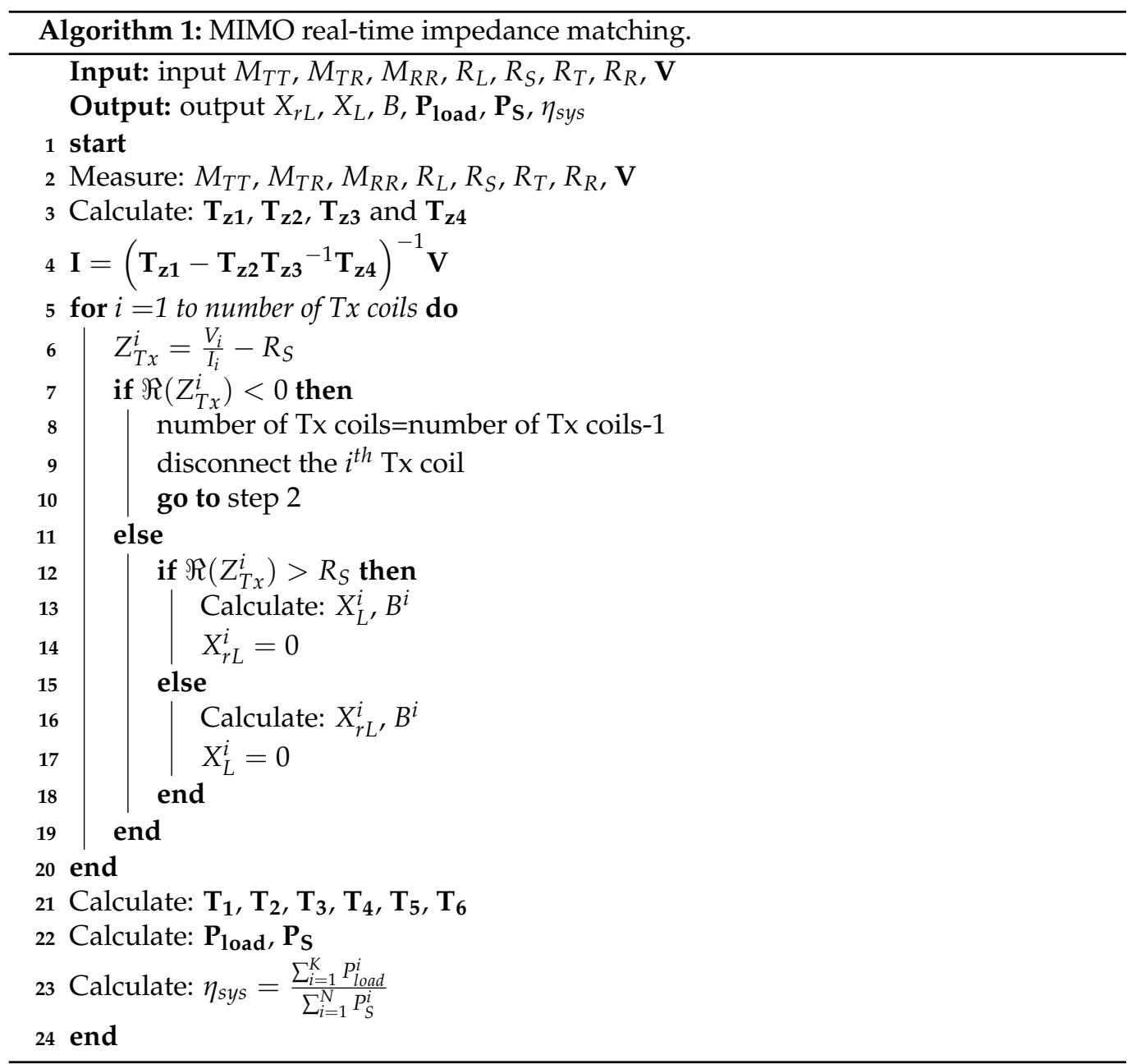




\subsection{Measuring the Coupling Inductance M between the Calculation Coils}

The first step is to measure the coupling inductance $M$ between the calculated coils. The coupling inductance $M_{T T}$ between the transmitting coil and the coupling inductance $M_{R R}$ between the receiving coil are measured and analyzed by measurement component. Turning on each Tx coil and Rx coil in sequence, we can obtain the coupling inductance between each $T x$ coil and Rx coil $M_{T R}$.

\subsection{Tx Impedance Measurement}

The second step is to measure and calculate the input impedance. The calculation of the input impedance requires the current went through the transmitter coil and the voltage at the right end of the impedance matching network. The expression of the relationship between current and voltage is shown in Equation (5). Using Ohm's law, we can obtain the input impedance, which is shown in Equation (7). In practice, we can use the AD8302 amplitude \& phase measurement monolithic integrated circuit and a microcontroller to form a detection circuit to achieve automatic detection and calculation of the input impedance.

\subsection{Selection of Transmitter}

The third step is the selection of transmitter Tx. The transmitting coils will be coupled with each other, which will cause corresponding effects. If the transmitting coils are close to each other, the energy transmitted from the transmitting coils may be smaller than the energy received, which causes waste to the energy transmission. For this problem, we adopt the scheme of transmitter Tx selection. Using Equation (7) to obtain the input impedance, if the real part of the input impedance $\Re\left(Z_{\text {in }}\right)<0$, micro-controller will disconnect the coil by using swithes until the real part of the input impedance $\Re\left(Z_{\text {in }}\right)>0$ for all coils.

\subsection{IMN-Type Decision and Respective Component Values}

The final step is to determine the type of IMN with the calculation of the values for each component. As a real-time inpedance matching network, the value of each component will change every $T$ seconds. According to Equation (16), the equivalent impedance of the right side of the IMN $Z_{T x}$ can be obtained. When $\Re\left(Z_{T x}\right)>R_{S}$, shorting $X_{R L}$, the IMN behaves as an L-type IMN, and the IMN component parameters can be calculated as in Equation (17). Similarly, when $\Re\left(Z_{T x}\right)<R_{S}$, shorted to $X_{L}$, IMN behaves as an reverse L-type IMN, and IMN element parameters can be calculated as in Equation (18). In real applications, this step is implemented within the micro-controller. Micro-controller calculates capacitance values $(C)$ and inductance values $\left(L_{L}\right.$ and $\left.L_{R L}\right)$ for capacitor matrix $(B)$ and inductor matrices $\left(X_{L}\right.$ and $\left.X_{R L}\right)$. The micro-controller will control IMNs by using SMPTs.

\section{Simulation and Analysis}

\subsection{System Set-Up}

In this paper, we take a 3-to-3 CMR system as an example. In the 3-to-3 CMR system, there are 3 Tx coils and 3 Rx coils. Each Tx coil connect with IMN and AC power source. Totally, there are 3 transmitters, $3 \mathrm{AC}$ power sources, $3 \mathrm{IMN}$ and 3 receivers in the 3 -to-3 CMR system. The power supplies provide the energy for the CMR system; the IMN system achieves impedance matching to improve power transfer efficiency and received power; and the receivers receive the electromagnetic waves and supply energy to the load devices. The critical parameters of the WPT system can be seen in Table 1. 
Table 1. Critical parameters of the WPT system.

\begin{tabular}{cc}
\hline Parameters & Value \\
\hline Size of TX coil & $7 \times 0.05 \mathrm{~m}$ \\
Equivalent inductance of TX coil & $7.175 \mu \mathrm{H}$ \\
Series capacitor for TX coil & $76.8 \mathrm{pF}$ \\
Size of RX coil & $7 \times 0.05 \mathrm{~m}$ \\
Equivalent inductance of RX coil & $7.175 \mu \mathrm{H}$ \\
Series capacitor for RX coil & $76.8 \mathrm{pF}$ \\
Operating frequency of power source & $6.78 \mathrm{MHz}$ \\
Impedance of the power source & $50 \Omega$ \\
Voltage of the power source & $15 \mathrm{~V}$ \\
Load impedance & $50 \Omega$ \\
\hline
\end{tabular}

In the simulation, we lined up coils separately. Figure 7 shows the schematic figure of coil placement. The adjacent Tx coils are spaced $0.2 \mathrm{~m}$ apart. The $\mathrm{Rx}$ coils are same as Tx coils. The distance of Tx coils and Rx coils was moved from $0.001 \mathrm{~m}$ to $0.5 \mathrm{~m}$. To achieve real-time impedance matching, the system calculate and change the IMN per $T$ seconds. In this case, the system can react actively to the distance between each coils.

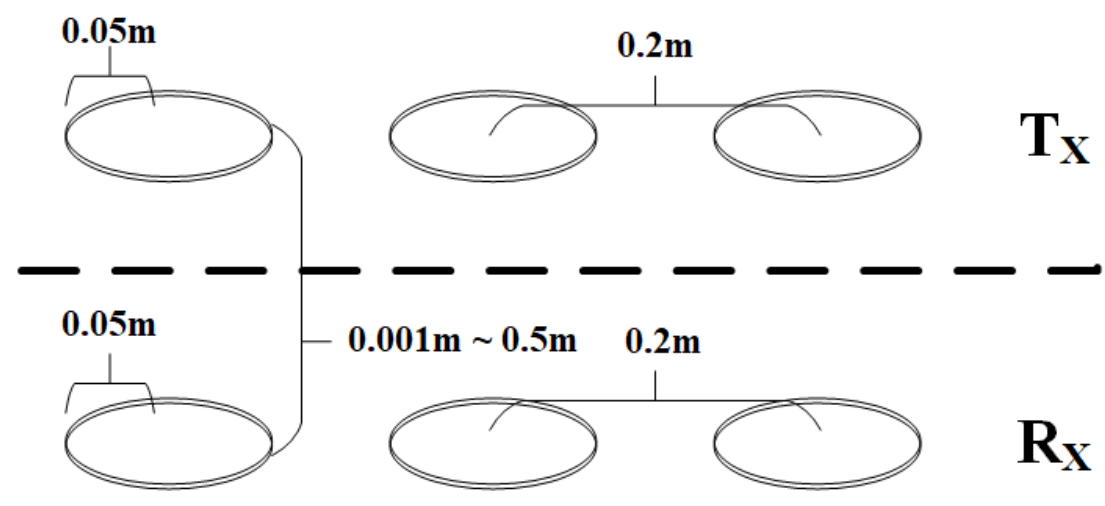

Figure 7. Schematic figure of coils placement.

\subsection{IMN Components}

The IMN which consists two inductance matrices and one capacitance matrix. In practical situation, the component matrix represents that $B, X_{R L}$ and $X_{L}$ will change in a discrete form. For the best performance of the simulated system, we assume that the inductance matrices and capacitance matrix have infinite size and number of components. Therefore $B, X_{R L}$ and $X_{L}$ will change in continuous form. Figure 8 shows the variation of IMN in different cases. The number and type of IMN will change in each time period, while the micro-controller detects that the $\Re\left(Z_{T x}\right)$ less than 0 , the micro-controller will disconnect that coil by using a switch. After selecting the coil, the micro-controller calculates the optimal values of capacitor matrix $(B)$ and inductor matrices $\left(X_{R L}\right.$ and $\left.X_{L}\right)$ based on the input impedance $Z_{i n}$. The type of IMN and the variation of component values are listed in Table 2. 


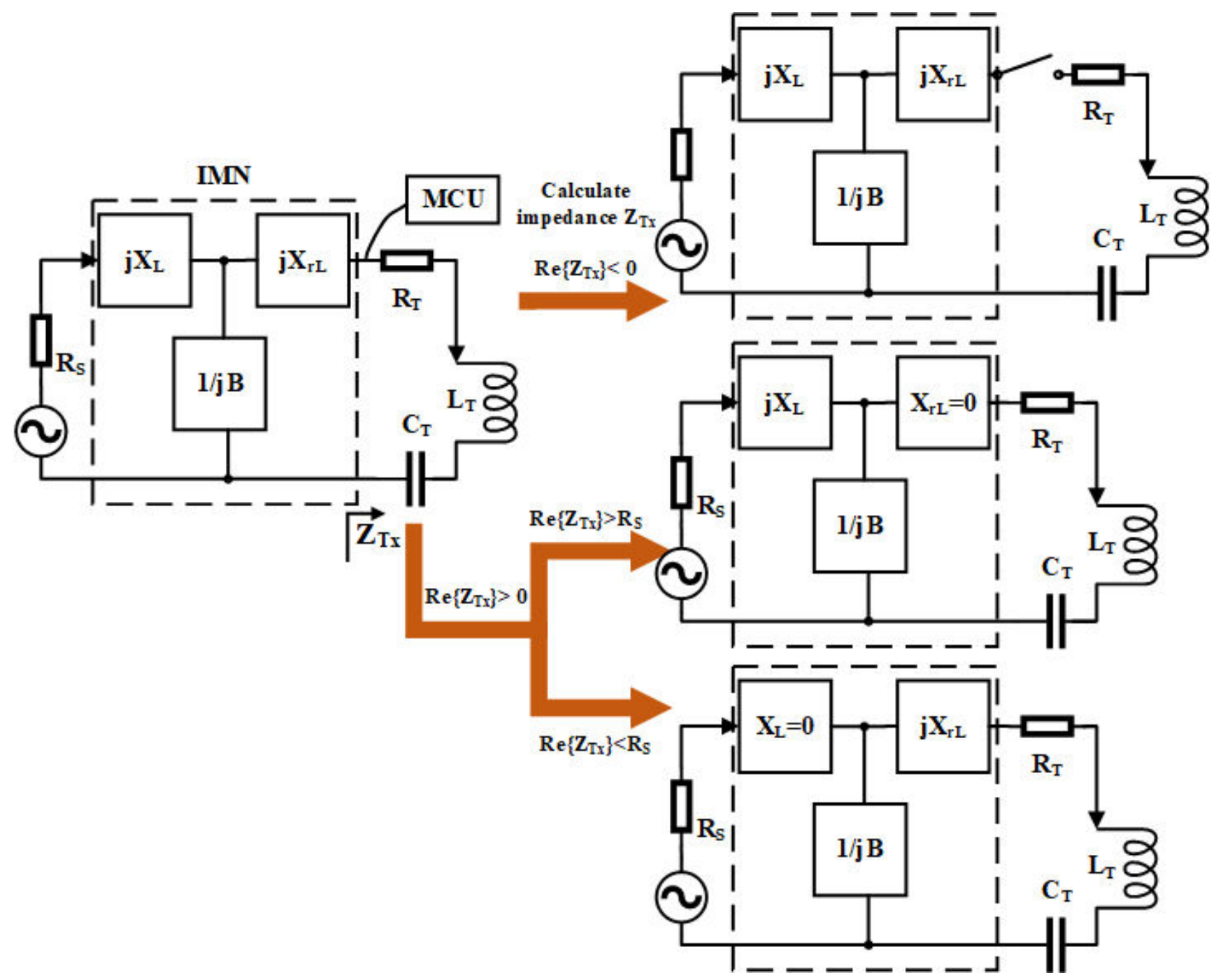

Figure 8. Variation of IMN in different cases.

Table 2. Components of the IMN.

\begin{tabular}{|c|c|c|c|c|c|c|c|c|}
\hline Distance & Impedance & IMN Type & B & $\mathbf{X}_{\mathrm{L}}$ & $X_{R L}$ & $\mathrm{C}(\mathrm{nH})$ & $\mathrm{L}_{\mathrm{L}}(\mu \mathrm{F})$ & $\mathrm{L}_{\mathrm{RL}}(\mu \mathrm{F})$ \\
\hline \multirow{3}{*}{0.001} & $5520-335 i$ & $\mathrm{~L}$ & 0.0019 & 523.9 & 0 & 0.28 & 77.27 & 0 \\
\hline & $5520-599 i$ & $\mathrm{~L}$ & 0.0019 & 526.3 & 0 & 0.28 & 77.76 & 0 \\
\hline & $5520-335 i$ & $\mathrm{~L}$ & 0.0019 & 523.9 & 0 & 0.28 & 77.27 & 0 \\
\hline \multirow{3}{*}{0.1} & $6.04+2.59 i$ & Reverse L & 0.0539 & 0 & 13.7 & 7.94 & 0 & 2.03 \\
\hline & $6.77+4.96 i$ & Reverse L & 0.0505 & 0 & 12.2 & 7.44 & 0 & 1.79 \\
\hline & $6.04+2.59 i$ & Reverse L & 0.0539 & 0 & 13.7 & 7.94 & 0 & 2.03 \\
\hline \multirow{3}{*}{0.2} & $0.51+3.07 i$ & Reverse L & 0.196 & 0 & 1.97 & 28.9 & 0 & 0.15 \\
\hline & $0.22+5.39 i$ & Reverse L & 0.2973 & 0 & 1.04 & 43.85 & 0 & 0.24 \\
\hline & $0.51+3.07 i$ & Reverse L & 0.196 & 0 & 1.97 & 28.9 & 0 & 0.15 \\
\hline \multirow{2}{*}{0.3} & $0.084+0.382 i$ & Reverse L & 0.4878 & 0 & 1.66 & 71.94 & 0 & 0.24 \\
\hline & $0.084+0.382 i$ & Reverse L & 0.4878 & 0 & 1.66 & 71.94 & 0 & 0.24 \\
\hline \multirow{2}{*}{0.4} & $0.06+0.38 i$ & Reverse L & 0.5844 & 0 & 1.32 & 86.19 & 0 & 0.19 \\
\hline & $0.06+0.38 i$ & Reverse L & 0.5844 & 0 & 1.32 & 86.19 & 0 & 0.19 \\
\hline \multirow{2}{*}{0.5} & $0.05+0.38 i$ & Reverse L & 0.6153 & 0 & 1.24 & 90.75 & 0 & 0.18 \\
\hline & $0.05+0.38 i$ & Reverse L & 0.6153 & 0 & 1.24 & 90.75 & 0 & 0.18 \\
\hline
\end{tabular}

The capacitance value $C$ of capacitor matrix $B$ can be reached by dividing $B$ by angular frequency $w$. Similarly the inductance value $L$ of inductor matrix can be reached by 
dividing $X$ by angular frequency $w$. The ranges of $C, X_{L}$ and $X_{R L}$ are commonly used in electronic devices. From the application point of view, the use of simple and inexpensive components to achieve optimal transmission efficient and transmission energy is feasible and effective.

\subsection{Result}

Table 3 illustrates the received power and power transfer efficiency of 3-to-3 CMR system before and after impedance matching. The third column of Table 3 is compared with the fifth column to obtain Figure 9. Figure 9 shows the power transfer efficiency of the SISO CMR system and the 3-to-3 CMR system before and after impedance matching. Comparing the second column of Table 3 with the fourth column, Figure 10 is obtained. Figure 10 shows the received power comparison between SISO CMR system and 3-to-3 CMR system before and after impedance matching. Divide the received power and power transfer efficiency of Table 3 to get Figure 11.

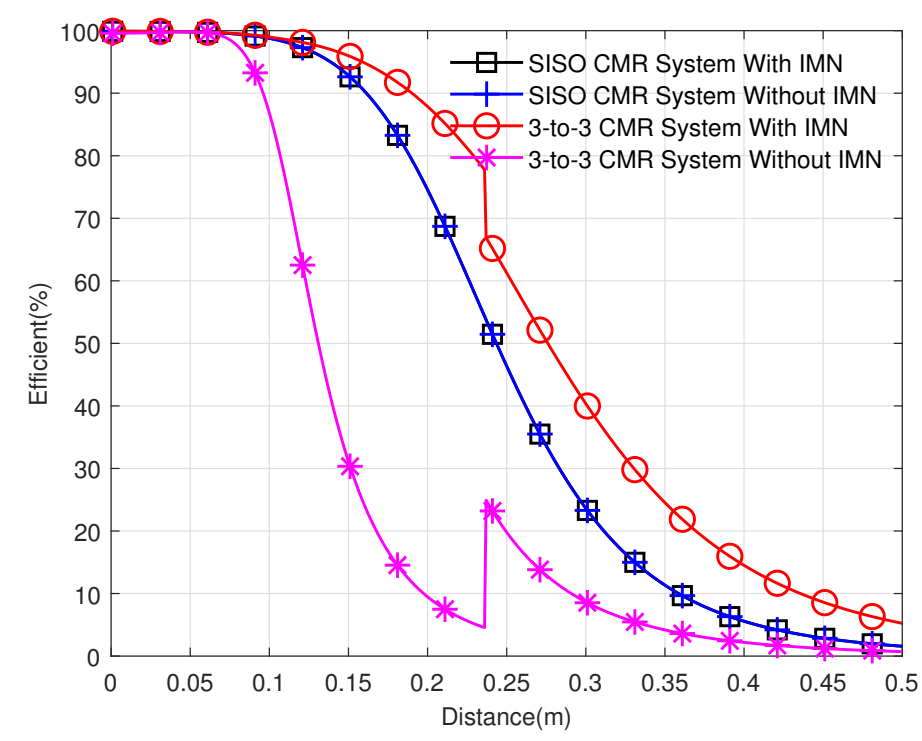

Figure 9. Power efficiency evaluations.

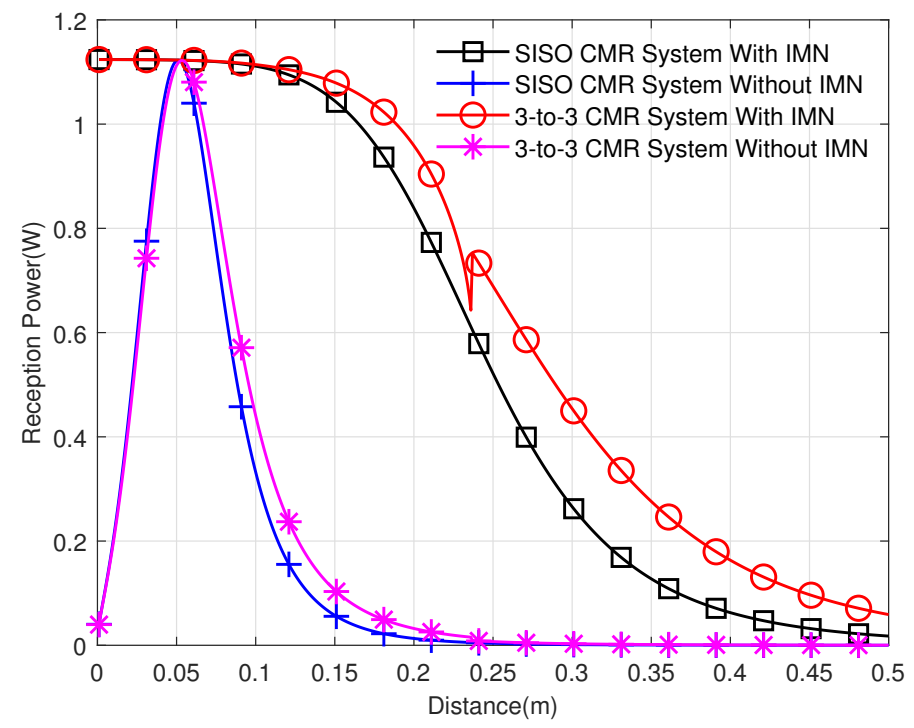

Figure 10. Received power evaluations. 


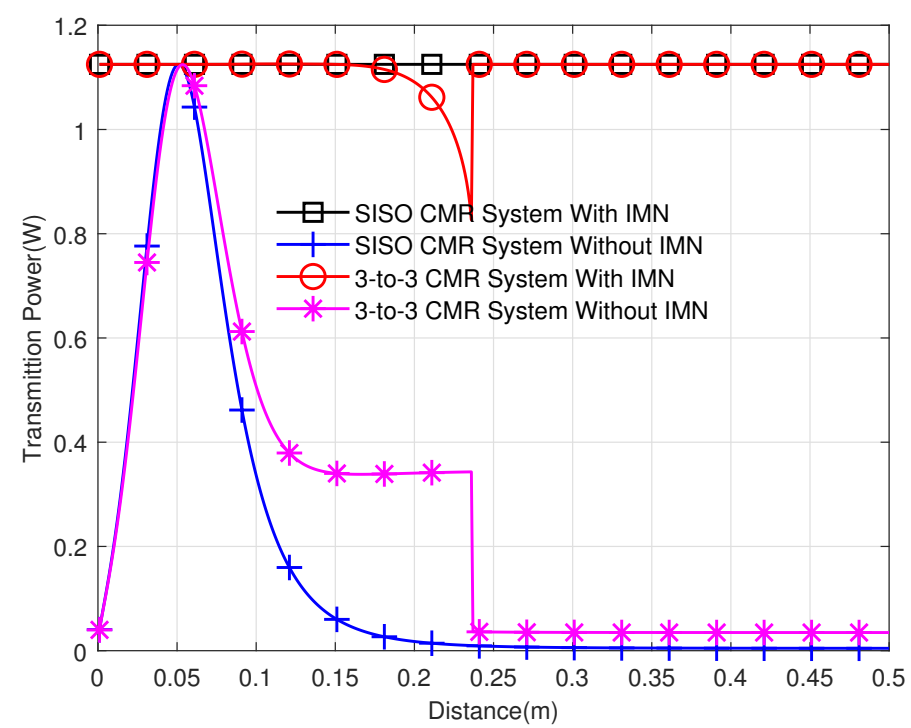

Figure 11. Source output power evaluations.

Table 3. Comparison of measured results with/without impedance matching.

\begin{tabular}{ccccc}
\hline Distance $(\mathbf{m})$ & \multicolumn{2}{c}{ Without IMN } & \multicolumn{2}{c}{ With IMN } \\
\hline & $(\mathrm{W})$ & Efficiency $(\%)$ & $(\mathrm{W})$ & Efficiency (\%) \\
0.001 & 0.04 & 99.59 & 1.24 & 99.89 \\
0.1 & 0.44 & 86.84 & 1.11 & 98.98 \\
0.2 & 0.032 & 9.47 & 0.96 & 87.86 \\
0.3 & 0.003 & 8.66 & 0.454 & 40.36 \\
0.4 & 0.00076 & 2.19 & 0.163 & 14.51 \\
0.5 & 0.00025 & 0.71 & 0.059 & 5.24 \\
\hline
\end{tabular}

As shown in Figures 9-11, the power transfer efficiency of the 3-to-3 CMR system was improved after impedance matching, while the power transfer efficiency of the 3-to-3 CMR system decreased without impedance matching. At the distance of $0.05 \mathrm{~m}$, the input impedance $Z_{i n}$ equal to internal resistance $R_{T}$ of power source. Therefore, both power and transfer efficiency reach its maximum. We notice that 3-to-3 MIMO system drops when the distance near $0.24 \mathrm{~m}$. That is because that when the dianstance between Tx coils and $\mathrm{Rx}$ coils at medium and long distances, the coupling between the transmitting coils dominates. It will reflect that received power of Tx coil is greater than the transmitted power. Because of the conflict between received power and transmitted power, we can noticed that received power and transmitted power decrease sharply at the distance of $0.2 \mathrm{~m}$. Consequently, the MIMO real-time impedance matching algorithm automatically disconnects this coil to optimize the system transmission power. By coil selecting, the received power and transmitted power of 3-to-3 MIMO CMR system will increase. The reason for the decrease in energy transmission efficient is that the MIMO system changes from three Tx coils to two Tx coils. The conversion of electromagnetic waves in space into electrical energy is less efficient. Therefore, the energy transfer efficient decreases at $0.24 \mathrm{~m}$ in Figure 9. When the distance between the Tx coils and Rx coils equal to $0.236 \mathrm{~m}$, the power transfer efficiency of 3-to-3 MIMO CMR system with IMN is $23.5 \%$ higher than SISO CMR system without IMN.

\section{Conclusions}

In this paper, we focus on the analysis and construction of a MIMO CMR system model. This model can be used to simulate and analyze the state of the MIMO CMR system before and after impedance matching. Based on the MIMO CMR system model, we propose an adaptive impedance matching scheme for magnetic MIMO wireless power transfer system. For every $T$ seconds, IMN will change according to the distance. In this 
case, the system can react accurately to the distance between coils. By using MIMO realtime impedance matching algorithm, the proposed scheme is able to improve impedance mismatching problem. The simulation result indicate that MIMO CMR system with IMN and MIMO real-time impedance matching algorithm can achieve higher energy transfer power and efficiency than MIMO CMR systems without IMN.

Author Contributions: Conceptualization of MIMO CMR with IMN, Y.Z., Z.L. and D.L.; model design and analyze, Z.L.; algorithm design, Z.L. and Y.Z.; simulations, Z.L. All authors have read and agreed to the published version of the manuscript.

Funding: This work was partially supported by National Nature Science Foundation of China (No. 61801306), Shenzhen Fundamental Research (No. JCYJ20180302145755311), Guangdong Special Fund for Science and Technology Development (No. 2019A050503001), the Open Research Fund of State Key Laboratory of Space-Ground Integrated Information Technology under grant NO. 2018 SGIIT KFJJ ST 01.

Data Availability Statement: No new data were created in this study. Data sharing is not applicable to this article.

Conflicts of Interest: The authors declare no conflict of interest.

\section{References}

1. Madawala, U.; Thrimawithana, D. A Bidirectional Inductive Power Interface for Electric Vehicles in V2G Systems. IEEE Trans. Ind. Electron. 2011, 58, 4789-4796. [CrossRef]

2. Kurs, A.; Karalis, A.; Moffatt, R.; Joannopoulos, J.D.; Fisher, P.; Soljacic, M. Wireless Power Transfer via Strongly Coupled Magnetic Resonances. Science 2007, 317, 83-86. [CrossRef] [PubMed]

3. Sasaki, S.; Tanaka, K.; Maki, K.i. Microwave Power Transmission Technologies for Solar Power Satellites. Proc. IEEE 2013, 101, 1438-1447. [CrossRef]

4. Jadidian, J.; Katabi, D. Magnetic MIMO: How to charge your phone in your pocket. In Proceedings of the 20th Annual International Conference on Mobile Computing and Networking ACM, Maui, HI, USA, 7-11 September 2014; pp. 495-506. [CrossRef]

5. Cheon, S.; Kim, Y.H.; Kang, S.Y.; Lee, M.L.; Lee, J.M.; Zyung, T. Circuit-Model-Based Analysis of a Wireless Energy-Transfer System via Coupled Magnetic Resonances. IEEE Trans. Ind. Electron. 2011, 58, 2906-2914. [CrossRef]

6. Karalis, A.; Joannopoulos, J.; Soljačić, M. Efficient wireless non-radiative mid-range energy transfer. Ann. Phys. 2008, 323, 34-48. [CrossRef]

7. Jiang, H.; Zhang, J.; Lan, D.; Chao, K.K.; Liou, S.; Shahnasser, H.; Fechter, R.; Hirose, S.; Harrison, M.; Roy, S. A Low-Frequency Versatile Wireless Power Transfer Technology for Biomedical Implants. IEEE Trans. Biomed. Circ. Syst. 2013, 7, 526-535. [CrossRef] [PubMed]

8. Choi, S.Y.; Gu, B.W.; Jeong, S.Y.; Rim, C.T. Advances in Wireless Power Transfer Systems for Roadway-Powered Electric Vehicles. IEEE J. Emerg. Sel. Top. Power Electron. 2015, 3, 18-36. [CrossRef]

9. Nguyen, M.Q.; Chou, Y.; Plesa, D.; Rao, S.; Chiao, J.C. Multiple-Inputs and Multiple-Outputs Wireless Power Combining and Delivering Systems. IEEE Trans. Power Electron. 2015, 30, 6254-6263. [CrossRef]

10. Aoki, T.; Yuan, Q.; Quang-Thang, D.; Okada, M.; Hsu, H.M. Maximum Transfer Efficiency of MIMO-WPT System. In Proceedings of the 2018 IEEE Wireless Power Transfer Conference (WPTC), Montreal, QC, Canada, 3-7 June 2018; pp. 1-3. [CrossRef]

11. Lang, H.D.; Ludwig, A.; Sarris, C.D. Convex Optimization of Wireless Power Transfer Systems With Multiple Transmitters. IEEE Trans. Antennas Propag. 2014, 62, 4623-4636. [CrossRef]

12. Luo, Y.; Yang, Y.; Chen, Z. Self-tuning Wireless Power Transmission Scheme Based on On-line Scattering Parameters Measurement and Two-side Power Matching. Sci. Rep. 2014, 4, 4332. [CrossRef] [PubMed]

13. Liu, J.; Zhao, Y.; Xu, C.Z.; Wang, X. One-side automated discrete impedance matching scheme for wireless power transmission. In Proceedings of the 2017 IEEE Wireless Power Transfer Conference (WPTC), Taipei, Taiwan, 10-12 May 2017; pp. 1-4. [CrossRef]

14. Kim, J.; Son, H.C.; Kim, D.H.; Park, Y.J. Impedance matching considering cross coupling for wireless power transfer to multiple receivers. In Proceedings of the 2013 IEEE Wireless Power Transfer (WPT), Perugia, Italy, 15-16 May 2013; pp. 226-229. [CrossRef]

15. Lee, J.; Lim, Y.; Ahn, H.; Yu, J.D.; Lim, S.O. Impedance-Matched Wireless Power Transfer Systems Using an Arbitrary Number of Coils With Flexible Coil Positioning. IEEE Antennas Wirel. Propag. Lett. 2014, 13, 1207-1210. [CrossRef]

16. Li, Y.; Dong, W.; Yang, Q.; Zhao, J.; Liu, L.; Feng, S. An Automatic Impedance Matching Method Based on the FeedforwardBackpropagation Neural Network for a WPT System. IEEE Trans. Ind. Electron. 2019, 66, 3963-3972. [CrossRef]

17. Jeong, S.; Lin, T.H.; Tentzeris, M.M. A Real-Time Range-Adaptive Impedance Matching Utilizing a Machine Learning Strategy Based on Neural Networks for Wireless Power Transfer Systems. IEEE Trans. Microw. Theory Tech. 2019, 67, 5340-5347. [CrossRef] 
18. Zhai, B.; Yang, M.; Liu, G. Trim method of impedance matching for magnetic resonance coupling system. In Proceedings of the 2017 29th Chinese Control Furthermore, Decision Conference (CCDC), Chongqing, China, 28-30 May 2017; pp. $2157-2161$. [CrossRef]

19. Kim, N.Y.; Kim, K.Y.; Ryu, Y.H.; Choi, J.; Kim, D.Z.; Yoon, C.; Park, Y.K.; Kwon, S. Automated adaptive frequency tracking system for efficient mid-range wireless power transfer via magnetic resonanc coupling. In Proceedings of the 2012 42nd European Microwave Conference, Amsterdam, The Netherlands, 29 October-1 November 2012; pp. 221-224. [CrossRef]

20. Lee, W.T.; Kung, M.L.; Lin, K.H. $\pi$-Impedance Matching Circuit for Wireless Power Transfer Systems under Dynamic Load and Transfer Distance. In Proceedings of the 2020 IEEE International Symposium on Antennas and Propagation and North American Radio Science Meeting, Montreal, QC, Canada, 5-10 July 2020; pp. 1579-1580. [CrossRef]

21. Lu, Y.; Mai, S.; Zhang, C.; Chen, H.; Wang, Z. Design optimization of printed spiral coils and impedance matching networks for load-variable wireless power transfer systems. In Proceedings of the 2017 International Conference on Electron Devices and Solid-State Circuits (EDSSC), Hsinchu, Taiwan, 18-20 October 2017; pp. 1-2. [CrossRef]

22. Fujiki, K.; Ishihara, M.; Umetani, K.; Hiraki, E. Experimental Verification of Impedance Matching Method for Repeater to Improve Spatial Freedom of 6.78 MHz Resonant Inductive Coupling Wireless Power Transfer Systems. In Proceedings of the 2019 21st European Conference on Power Electronics and Applications (EPE '19 ECCE Europe), Genova, Italy, 3-5 September 2019; pp. P.1-P.10. [CrossRef]

23. Boo, S.H.; Lee, B. Maximum WPT Efficiency Using Adaptive Impedance Matching. In Proceedings of the 2018 International Symposium on Antennas and Propagation (ISAP), Busan, Korea, 23-26 October 2018; pp. 1-2. 